\title{
Germanica
}

\section{E.R. Curtius et H.G. Gadamer ou la rencontre de la philologie et de la philosophie}

E.R.Curtius und H.G.Gadamer oder die Begegnung von der Philologie und der Philosophie

\section{Christine Jacquemard-de Gemeaux}

\section{(2) OpenEdition}

Journals

Édition électronique

URL : http://journals.openedition.org/germanica/2405

DOI : 10.4000/germanica.2405

ISSN : 2107-0784

\section{Éditeur}

Université de Lille

\section{Édition imprimée}

Date de publication : 30 juin 2000

Pagination : $33-43$

ISBN : 9782913857032

ISSN : 0984-2632

Référence électronique

Christine Jacquemard-de Gemeaux, «E.R. Curtius et H.G. Gadamer ou la rencontre de la philologie et de la philosophie », Germanica [En ligne], 26 | 2000, mis en ligne le 07 mars 2014, consulté le 06 octobre 2020. URL : http://journals.openedition.org/germanica/2405; DOI : https://doi.org/10.4000/ germanica.2405

Ce document a été généré automatiquement le 6 octobre 2020.

(c) Tous droits réservés 


\title{
E.R. Curtius et H.G. Gadamer ou la rencontre de la philologie et de la philosophie
}

\author{
E.R.Curtius und H.G.Gadamer oder die Begegnung von der Philologie und der \\ Philosophie
}

Christine Jacquemard-de Gemeaux

1 Curtius et Gadamer se sont rencontrés ${ }^{1}$ au cours des années 1920, à Marbourg, où l'un professait déjà, alors que l'autre commençait tout juste ses études. Dans ses mémoires, Gadamer rapporte :

Durch Ernst Robert Curtius, mit dem ich persönlich einen für mich ehrenvollen und gewinnbringenden Umgang hatte, wurde ich damals als Zwanzigjähriger mit Max Scheler zusammengeführt [...] Es gehörte zu meinen schönen Vorrechten, daß ich als Student manchmal nachmittags Ernst Robert Curtius zu einem Spaziergang abholen durfte $[. . .]^{2}$.

2 Et de relater la stimulation intellectuelle qu'il retire de ces précieux moments. Pourtant, seize années séparent les deux hommes et, à l'époque, il ne peut être question d'un véritable dialogue entre eux. L'œuvre du premier s'achève au début des années 1950, après les succès remportés par Europäische Literatur und lateinisches Mittelalter ${ }^{3}$ et les Kritische Essays zur europäischen Literatur ${ }^{4}$, tandis que l'opus magnum du second Wahrheit und Methode ${ }^{5}$ - n'est publié que dix années plus tard, en 1960, alors que Curtius est déjà décédé.

3 À première vue, leur horizon intellectuel diffère également: l'un est philologue, romaniste, formé à l'école strasbourgeoise de Gustav Gröber ; l'autre est philosophe, élève du néokantien Paul Natorp à Marbourg, puis de Heidegger à Heidelberg. Mais on oublie fréquemment que Gadamer reçut d'abord une solide formation de philologue ${ }^{6}$ et que Curtius, lorsqu'il était étudiant, suivit avec enthousiasme ${ }^{7}$ les cours d'un autre néokantien, Wilhelm Windelband ${ }^{8}$, à Heidelberg précisément. Un faisceau de convergences apparaît de fait distinctement lorsqu'on examine leurs démarches et leurs œuvres. Ni Gadamer ni Curtius ne se cantonnent dans les limites de leur 
spécialité. En esprits universels, ils établissent des passerelles entre différents champs d'investigation. Et surtout, tous deux sont représentatifs d'une des grandes orientations de la pensée au $\mathrm{XX}^{\mathrm{e}}$ siècle : l'intérêt porté à la langue et au langage. Face à la crise intellectuelle, ils placent ces domaines et, en fin de compte, la littérature au centre de la réflexion. Dépassant la rivalité qui oppose littérature et philosophie afin d'expliquer le monde, ils tentent de faire comprendre la liaison qui se fait jour entre ces deux espaces, dans les pays de langue allemande, au cours du XXe siècle, et surtout quels en sont les enjeux.

Dans le domaine de l'esprit, le constat que font les intellectuels dès le début du siècle, et tout particulièrement après la catastrophe de la Grande Guerre, est celui d'un ébranlement général de la pensée. Ainsi Hofmannsthal écrit-il: "Es gibt nichts im geistigen Bereich, das nicht versehrt wäre $[. ..] »^{9}$. Certes la modernité n'est pas morte, mais elle se trouve remise en question; sécrétant ses propres contradictions, elle cesse progressivement de déterminer le paradigme de l'époque, tout comme la philosophie cesse d'être la reine des sciences. L'acte spéculatif pur a perdu de son importance. Curtius rappelle la situation par ces mots : " Als Hegel schrieb, stand die philosophische Spekulation Deutschlands im Zenith [...] Sie erhob die Geschichte zur Würde des Geistes, aber annektierte sie zugleich [...] auf das philosophische folgte das historische Zeitalter $\aleph^{10}$. Il évoque ainsi le passage de la philosophie à la philosophie de l'histoire, puis à l'historisme qu'il ne définit pas comme une théorie scientifique, mais comme une forme de point de vue de l'esprit, une "Weltanschauung " qui aurait vu le jour dans l'espace intellectuel de Hegel et de Ranke, de Nietzsche et de Jakob Burckhardt ${ }^{11}$. Au cours du XIX ${ }^{\mathrm{e}}$ siècle, cet historisme affirme son hégémonie en même temps qu'il évolue vers un système clos, un relativisme total, dont la modernité se montre incapable de surmonter les apories; les seules catégories rationnelles ne suffisent plus à sortir de l'impasse. Dès lors, la question fondamentale et pressante devient, ou redevient, celle $d u$ sens et de la recherche du sens. Comment trouver encore non pas le sens mais du sens ? Comment approcher une ou des vérités?

5 Avec Dilthey, Husserl, Heidegger et finalement Gadamer, la dynamique de l'herméneutique moderne permet d'ouvrir de nouvelles perspectives. Réexaminant la situation spécifique des sciences humaines, c'est dans le domaine de l'art que Gadamer engage sa quête de la vérité et sa critique de la modernité. L'art reste en effet le seul domaine qui échappe à la conception scientifique du monde ; c'est donc par lui que la réflexion sera en mesure de frayer une voie vers l'essentiel. Pour le philosophe comme pour le philologue, il ne s'agit pas de dénier toute valeur à la rationalité, ni même de négliger ses apports, mais de la dépasser. Le discours conceptuel ne suffisant plus, il s'impose de sortir de ses cadres. Et la littérature, à la fois art du langage et fonction discursive, semble justement permettre d'ouvrir cet espace où il est possible de poser à nouveau la question de l'être. Gadamer le situe à la frontière entre les sciences humaines et l'œuvre d'art.

6 Pour ces raisons, la littérature représente le lieu le plus stimulant pour la pensée, Gadamer en est convaincu : «Jedenfalls liegt im Phänomen der Literatur nicht zufällig der Punkt, an dem Kunst und Wissenschaft ineinander übergehen $»^{12}$. Le concept même de littérature doit être entendu au sens large - « der Begriff der Literatur [ist] sehr viel weiter als der Begriff des literarischen Kunstwerks $»^{13}$ - il recoupe l'ensemble de toutes les formes possibles du discours: littérature, poésie, éloquence, textes religieux, juridiques et finalement toutes les sciences humaines («[...] mithin das Ganze der 
Geisteswissenschaften $\left.»^{14}\right)$. La littérature a un caractère englobant : elle n'est pas une poésie qui aurait perdu « sa valence ontologique ${ }^{15}$, c'est pourquoi elle apparaît comme l'objet de choix du philologue et du philosophe. Dans le domaine de la critique littéraire, Curtius se fait, suivant le sillage de Dilthey, un des meilleurs représentants du courant herméneutique. Empruntant des chemins différents, Curtius et Gadamer aboutissent à des conclusions étonnamment similaires.

7 Le premier problème rencontré par les deux chercheurs, celui qui éclaire et guide leur réflexion, est celui de la méthode. En tentant de le résoudre, Curtius et Gadamer effectuent une remise en cause de l'esthétique moderne telle qu'elle naquit au siècle des Lumières avec Kant. Ils cherchent la vérité de l'art qui «a été oubliée et effacée par tout un ensemble de constructions métaphysiques nées avec la modernité et qui, à partir du milieu du XVIII ${ }^{e}$ siècle, ont pris le nom d'esthétique ${ }^{16}$. Au siècle suivant, le primat du scientisme positiviste fait obstacle à l'appréhension de la vérité. Ainsi que le souligne Marc Fumaroli :

Dans Vérité et méthode, Gadamer a établi, dans une ligne de pensée qui remonte à Dilthey, que la vérité humaine, distincte de la vérité scientifique, ne peut être atteinte par les techniques linéaires de la démonstration apodictique mais par les approximations de l'herméneutique, interlocutrice naturelle de la persuasion rhétorique et poétique ${ }^{17}$.

De fait, indique Gadamer dès les premières lignes de Wahrheit und Methode, la méthode inductive, appliquée avec succès aux sciences dites "exactes ", n'est pas valable pour les sciences humaines. Elle conduit à un positivisme stérilisant qui ne permet pas de dégager du sens. Le positiviste - A. Comte et J. S. Mill sont ici visés - ne cherche pas à connaître l'objet de son étude dans sa vérité mais uniquement à trouver les méthodes efficaces de son analyse scientifique. Utilisant exclusivement l'induction, avec pour objectif de dégager des lois et, partant, de prévoir des phénomènes («[...] Gleichförmigkeiten, Regelhaftigkeiten, Gesetzmäßigkeiten $\mathrm{zu}$ erkennen, die die einzelnen Erscheinungen und Abläufe voraussagbar [machen] ») ${ }^{18}$, il échoue face à la réalité des "Geisteswissenschaften ». Car ce type de sciences, qui comprend aussi le droit et la théologie, ne se fonde pas sur l'étude d'éléments purement naturels mais sur celle de textes produits par l'esprit humain, qui relèvent donc du domaine de l'expérience ("Erfahrung», ou "Welterfahrung»). Et Gadamer de faire l'historique du mot "Geisteswissenschaften" - introduit en 1849 par le traducteur de J. S. Mill ${ }^{19}$ pour traduire l'expression anglaise "moral sciences»-: il montre que ce terme est indubitablement lié au vaste monde psychique de l'expérience.

Dans le domaine des sciences humaines, on peut certes repérer des lois internes à chaque œuvre, des lois uniques, mais il n'en existe pas de générale, applicable à l'ensemble des textes. Lorsqu'on prétend les analyser comme des sciences exactes ( [ [...] wenn man sie an dem Maßstab fortschreitender Erkenntnis von Gesetzmäßigkeit mißt » $)^{20}$, lorsqu'on cherche à leur appliquer une grille d'interprétation, on n'aboutit à aucun résultat, on ne résout pas ce que Gadamer appelle "die Wahrheitsfrage » des sciences humaines. Dans le premier chapitre de Wahrheit und Methode, Gadamer affirme d'emblée son point de vue : "Es gibt keine eigene Methode der Geisteswissenschaften » ${ }^{21}$ et de se référer au physicien Helmholtz qui parle de « tact " par rapport au texte, tact qui remplacerait l'usage conscient de l'induction (« vom psychologischen Takt, der hier 
an die Stelle des bewußten Schließens trete ") $)^{22}$. Gadamer s'interroge à ce sujet, se demande comment on acquiert ce "tact», et laisse finalement entendre qu'il s'agit d'un don, fondement véritable de toutes les découvertes en sciences humaines, don qui se manifeste dans le domaine de la littérature, de la critique littéraire, qu'il préfère pour sa part désigner par le terme plus adéquat d'interprétation (" Auslegung »). Interrogé sur son travail de critique littéraire, Curtius ne définit pas non plus de méthode ; il récuse d'ailleurs le terme. Selon lui, toutes les méthodes sont réductrices, qu'elles soient structuraliste, psychanalytique, sociologique ou autres, car elles n'appréhendent qu'un aspect de la réalité, qu'elles ont tendance à "enfler " exagérément. Préférant parler de sa démarche, Curtius défend une conception compréhensive, interprétative, herméneutique de la critique où la subjectivité, l'individualité du chercheur entre en jeu. Et de définir lui aussi la critique comme un don, « eine Begabung » qui s'attache à mettre au jour les points originaux de l'œuvre : « Kritische Begabung ist nichts anderes als die Fähigkeit, von solchen Einzelzügen frappiert zu werden $»^{23}$. Dans son étude sur Marcel Proust, il décrit un cheminement qui va de la réception à la compréhension, en passant par la perception et la "déconstruction»:

Die Ruhe und die Passivität des reinen Aufnehmens muß die Grundhaltung des Kritikers sein. Rezeption ist die Vorbedingung der Perzeption und diese führt zur Konzeption. Denn über die Wahrnehmung und Festlegung der Einzelzüge hinaus schreitet die Kritik in synthetischem Verfahren zur Rekonstruktion der geistigen Gesamthaltung des Autors ${ }^{24}$.

11 Dès ses premiers travaux, le livre sur $B_{a l z a c}{ }^{25}$ - son meilleur ouvrage de critique littéraire -, et l'essai sur Proust, Curtius cherche donc d'abord l'empathie ("Einfühlung ») la plus profonde, voire l'abandon au texte par « imprégnation » totale, avant d'analyser rigoureusement une œuvre. Pour expliciter ce mouvement, il utilise le terme évocateur de "coefficient affectif $»^{26}$ nécessaire à toute authentique compréhension, il parle d'intuition.

Mais il se tient aussi à l'affût des traits singuliers d'une œuvre; constructions syntaxiques, effets de style, motifs et images itératifs qui lui permettent de retrouver de façon synthétique «le mystère de l'originalité créatrice» («das Geheimnis der schöpferischen Originalität »). Ces traits originaux lui servent de points d'ancrage pour reconstruire ensuite ce qu'il avait d'abord "démonté ». Dans le cas de La Recherche, dont il est un des premiers lecteurs en Allemagne, il parvient, dès 1922, à anticiper les développements imaginés par Marcel Proust avant même la parution de Albertine disparue et du Temps retrouvé: Curtius trouve ainsi une première vérité de l'œuvre. Cela ne saurait pourtant suffire pour atteindre une compréhension universelle de la littérature ; une autre démarche est nécessaire.

Il faut dépasser le filtre du modernisme, tenter de remonter aux origines, et retrouver le fil de la tradition. Curtius parvient à cette conclusion après avoir repéré les liens formels qui unissent les œuvres littéraires par-delà les siècles : si elles exercent leur pouvoir sur nous c'est qu'elles utilisent des arguments itératifs, rhétoriques, profondément enracinés dans notre culture. C'est ainsi que l'herméneutique se découvre être - selon les termes de M. Fumaroli - «l'interlocutrice naturelle de la rhétorique ». Pour le philosophe qu'est Gadamer, notre époque a opéré une réduction de l'art et de la littérature en les concevant uniquement dans le sens d'une esthétique de l'Erlebnis, mais nous prenons conscience : 
daß dieses Zeitalter im Ganzen der Geschichte der Kunst und der Dichtung nur eine Episode ist. Die großartigen Forschungen zur Literaturästhetik des Mittelalters, die Ersnt Robert Curtius zusammengefaßt hat, geben davon eine gute Vorstellung. Wenn man über die Grenzen der Erlebniskunst hinauszublicken beginnt und andere Maßstäbe gelten läßt, öffnen sich neue weite Räume innerhalb der abendländischen Kunst, die von der Antike, bis zum Zeitalter des Barocks von durchaus anderen Wertmaßstäben beherrscht war als von dem der Erlebtheit [... $]^{27}$

Gadamer rappelle qu'au XVIII ${ }^{e}$ siècle poésie et rhétorique sont encore étroitement et harmonieusement liées. Mais l'émergence de la théorie du génie aurait séparé l'art et la littérature de la tradition, du contexte historique, et donc de la rhétorique en tant qu'héritage occidental le plus profond. De fait, la tradition rhétorique assurait la transmission de cet héritage, avec en son centre la littérature, à la fois trésor de la tradition et creuset de l'avenir. Cela permet de redéfinir sa véritable fonction :

Das Dasein von Literatur ist nicht das tote Überdauern eines entfremdeten Seins, das der Erlebniswirklichkeit einer späteren Zeit in Simultaneität gegeben wäre. Literatur ist vielmehr eine Funktion geistiger Bewahrung und Überlieferung und bringt daher in jede Gegenwart ihre verborgene Geschichte ein ${ }^{28}$.

15 Trois textes ${ }^{29}$ principaux portent témoignage de l'intérêt de Gadamer pour la rhétorique: Rhetorik, Hermeneutik und Ideologiekritik. Metakritische Erörterungen zu Wahrheit und Methode (1967) - le plus important - ; Rhetorik und Hermeneutik (1976); et Logik oder Rhetorik. Nochmals zur Frühgeschichte der Hermeneutik (1976). Mais il n'entre pas dans notre propos de les analyser systématiquement, car Gadamer s'y interroge principalement sur les origines de l'herméneutique philosophique, sur son histoire. Dans Die Ausdruckskraft der Sprache. Zur Funktion der Rhetorik für die Erkenntnis (1979) ${ }^{30}$, il reprend l'idée d'une liaison entre rhétorique et dialectique, rhétorique et connaissance, cette dernière restant l'objet final de ses interrogations.

Curtius, en revanche, place la rhétorique au cœur de ses recherches philologiques. A l'heure de sa maturité intellectuelle, il décide d'allier à l'empathie herméneutique une perspective rhétorique, qui s'appuie essentiellement sur les notions d'inventio et de memoria. Partant de l'inventio, dont il souligne toute l'importance - «Die Lehre von der Auffindung des Stoffes ist der wichtigste Teil $»^{31}-$, il indique une nouvelle démarche qui s'attache à repérer et analyser les topoi, les lieux communs, qu'il nomme les constantes de la littérature européenne. Il voit dans les topoi, les « lieux de découverte » ("Die Fundörter»), les manifestations permanentes de la littérature. Ces lieux constituent les preuves et les arguments du discours littéraire. Mais ils n'ont pas un caractère figé: ils restent sujets à variation, ils sont porteurs d'une profonde dynamique. Les lieux suscitent le discours, font naître l'écriture, tout en servant de « lieux de mémoire »: ils sont doubles et doublement positifs. En les examinant à la loupe, on saisit la tension qui sous-tend la littérature européenne dans sa synchronie et sa diachronie ; l'éternelle tension entre imitation et créativité.

17 Poussant plus loin que Gadamer la réflexion purement philologique, Curtius forge l'idée d'une topique historique qui analyserait les œuvres de la tradition et de la création européenne sous leur aspect topique et dans leur tension temporelle. La rhétorique et sa memoria illustrent cette tension, ce mouvement dynamique qui se cristallise dans la conversation, le dialogue que la littérature poursuit avec l'histoire. Pour Curtius, l'étude des textes, l'étude de leurs composantes et des constantes du discours, permet de mettre en évidence le mouvement dialectique - c'est-à-dire chez lui, « dialogique » - 
de la pensée. Littérature et philosophie se rejoignent pour comprendre et permettre le dialogue.

Dans les écrits de Curtius, comme de Gadamer, on constate une même présence, une même importance, de la métaphore dialogique. Gadamer voit fondamentalement dans la structure herméneutique un cercle qui se boucle dans la dialectique de la question et de la réponse. L'interprétation («Die Auslegung ») d'un texte est d'ailleurs selon lui :

[...] wie das Gespräch ein durch die Dialektik von Frage und Antwort geschlossener Kreis. Es ist ein echtes geschichtliches Lebensverhältnis, das sich im Medium der Sprache vollzieht und das wir daher auch im Falle der Auslegung von Texten ein Gespräch nennen können ${ }^{32}$.

Dans toute interprétation, il se produit pour Gadamer « une entrée en dialogue avec le texte » («Ein In-das-Gesprächkommen mit dem Text »33), ce qui signifie que d'objet le texte devient sujet du dialogue. Dans sa récente étude sur le sujet, Pascal Michon critique cette perspective gadamérienne - qui rejoint ici Heidegger - ; car elle attribue " aux textes et aux choses qui y sont dites une nature subjective [...]. Sa philologie se présente comme [...] une philologie anthropomorphique $»^{34}$. Le reproche qui est fait ici concerne l'aspect irrationnel de la pensée de Gadamer, qui ne va pas cependant jusqu'au «mysticisme» de la philosophie heideggérienne. Gadamer souligne essentiellement le dialogue avec la tradition, en ce sens il apparaît plus conservateur.

Curtius, lui-aussi, souligne ce dialogue, la conversation permanente entrenue avec les textes littéraires et entre les textes eux-mêmes, comme s'ils se répondaient, nous répondaient, au-delà des espaces et des temps. Il met au jour la conversation de la tradition et de la modernité, mais il débouche plus nettement sur une quête anthropologique. Chez Curtius, la mise en évidence d'une dynamique dialogique conduit globalement à montrer l'importance de la liberté humaine et la permanence d'un humanisme fondamentalement ouvert sur l'avenir. C'est pourquoi on peut qualifier sa pensée d'humanisme rhétorique. Une parole est échangée dans le dialogue avec les textes, et surtout les textes littéraires; cette parole n'est pas gratuite mais constitutrice de liberté, d'identité et finalement d'humanité. C'est elle qui donne à voir le sens de l'humain.

21 En philosophe, Gadamer s'intéresse également, pour la littérature, comme pour son interprétation, au primat intellectuel de la liberté et du jeu - aspect intellectuel que n'ignore naturellement pas Curtius, lorsqu'il affirme par exemple:» Kritik bleibt ja immer ein Wagnis [...] Sie ist ein Akt schöpferischer geistiger Freiheit». L'enjeu de la poésie, c'est-à-dire de la littérature au sens large, est d'être pour ces deux pensées « die objektive Aufdeckung der Welt $\aleph^{35}$. Gadamer trouve finalement en la littérature l'espace ontologique par excellence. Il atteint ainsi à une anti-esthétique qui permet un nouveau rapport du sujet au monde, qui autorise à jeter une passerelle entre le sujet et la nature, par l'exercice créateur du langage, de la langue, et sa réalisation dans la littérature. De ce fait, la littérature devient l'espace central où l'être affleure. Elle permet non seulement de retrouver un sens, mais de le faire advenir.

Il semble finalement étonnant que Gadamer ne cite presque pas Curtius, son ancien maître de Marbourg, dans Wahrheit und Methode. La position de la littérature dans la pensée des deux hommes, l'importance qu'ensemble ils accordent à la critique, à l'interprétation littéraire, sont absolument frappantes. Il n'est pas jusqu'à certaines images utilisées par Gadamer qui ne rappellent curieusement Curtius. Ainsi évoquant le passé, Gadamer parle-t-il avec lyrisme des vestiges du temps qui passe : 
Die Überreste vergangenen Lebens, Reste von Bauten, Werkzeuge, der Inhalt der Gräber sind verwittert [souligné par nous] durch die Stürme der Zeit, die über sie hinweggebraust sind ${ }^{36}$.

Ces images d'intempéries ravageuses sont exactement les mêmes que celles dont se sert Curtius pour évoquer le Vivarium de Cassiodore vers 540 - «Ein Bildungskloster an der verwitterten [souligné par nous] Römerstraße $\aleph^{37}-$; de telles similitudes ne manquent pas d'attirer l'attention des familiers de l'œuvre curtiusienne. Il semble qu'à maints niveaux une vraie rencontre se produise entre le philosophe et le philologue. Il serait naturellement erroné de vouloir amalgamer les œuvres de Curtius et de Gadamer. Peutêtre y aurait-il cependant lieu, dans une nouvelle analyse de la pensée de Gadamer, de s'interroger plus précisément sur sa filiation?

\section{NOTES}

1. Ch. Jacquemard-de Gemeaux : «Curtius in Marburg. Vom deutsch-französischen Vermittler zum Repräsentanten der longue durée », in Die Universität Marburg in den zwanziger Jahren, Actes du Colloque de Marbourg, octobre 1999 (à paraître), et monographie de Curtius : Ernst Robert Curtius (1886-1956). Origines et cheminements d'un esprit européen, Bern, Peter Lang, Collection Contacts, Études et Documents 43, 1998. Cf. également, H. G. Gadamer: Philosophische Lehrjahre: eine Rückschau, Frankfurt am Main, V. Klostermann, 1995.

2. . Cf., Philosophische Lehrjahre, p. 26-27.

3. . E.R. Curtius : Europäische Literatur und lateinisches Mittelalter, Bern, Francke Verlag, 1948. J'utiliserai la dixième réédition de 1984, désormais citée comme ELLMA.

4. . E.R. Curtius: Kritische Essays zur europäischen Literatur [désormais: KEEL], Bern, Francke Verlag, 1950.

5. . J'utiliserai H.G. Gadamer : Hermeneutik II. Wahrheit und Methode, Tübingen, J.C.B. Mohr (Paul Siebeck), Tübingen, 1990. Certaines citations seront empruntées à Pascal Michon: Conditions théoriques d'une histoire du sujet. Séminaire 1997-1998. Trois questions à l'herméneutique [désormais : Conditions théoriques d'une histoire du sujet], Les Papiers du Collège International de Philosophie, $n^{\circ}$ 44, Paris, 1998 et réédition 1999.

6. . Cf., Philosophische Lehrjahre, p. 34 : «Es waren Jahre eines tiefen Zweifels an meiner wissenschaftlichen Begabung [...] Ich wurde klassischer Philologe unter der freudschaftlichen Anleitung von Paul Friedländer ».

7. . Gundolf Briefe, hrsg. von L. Helbing und Cl. Bock, Amsterdam, 1965, p. 99.

8. . C'est par deux fois, en été 1910 puis durant l'hiver 1911-1912, qu'il participa avec ferveur au séminaire de philosophie de Wilhelm Windelband (1848-1915), fondateur de l'école néokantienne dite « de Bade».

9. . « Vermächtnis der Antike », in : Insel Almanach auf das Jahr 1928, p. 58.

10. . E.R. Curtius, « Toynbees Geschichtslehre » in : KEEL, p. 347.

11. . «Der Historismus und seine Probleme», in : E. R. Curtius : Büchertagebuch, Bern, Francke Verlag, 1960, p. 99.

12. . Wahrheit und Methode, p. 168.

13. . Wahrheit und Methode, p. 167.

14. Ibid. 
15. . Wahrheit und Methode, p. 165 : « die ihrer Valenz entfremdete Poesie ».

16. Conditions théoriques d'une histoire du sujet, p. 4.

17. . Histoire de la rhétorique dans l'Europe moderne. 1450-1950, M. Fumaroli (Dir.), Paris, PUF, 1999, p. 1291.

18. . Wahrheit und Methode, p. 10.

19. . J. S. Mill : System der deduktiven und induktiven Logik, übertragen von Schiel, 1863, 6. Buch : «Von der Logik der Geisteswissenschaften oder moralischen Wissenschaften ». Sylvie Mesure, in: Dilthey et la fondation des sciences historiques, Paris, PUF, 1990, p. 56, précise que G. Droysen avait déjà utilisé l'expression en 1843 dans sa Geschichte des Hellenismus.

20. . Ibid.

21. . Wahrheit und Methode, p. 13.

22. . Ibid.

23. . E.R. Curtius: Marcel Proust, in: Französischer Geist im neuen Europa, Stuttgart, Deutsche Verlagsanstalt, 1925, réédition in : Französischer Geist im zwangzigsten Jahrhundert, Bern, Francke Verlag, 1952, p. 14.

24. Ibid.

25. . Balzac, Bonn, F. Cohen, 1923. ; Bern, Francke Verlag, 1951 ; Frankfurt/M., Fischer TB-Verlag, 1985.

26. . «Eine neue Geschichte der Mittellateinischen Literatur», in: E.R. Curtius : Gesammelte Aufsätze zur romanischen Philologie, Bern, Francke Verlag, 1950, 1984, p. 53. Curtius emprunte ce terme au livre de J. de Ghellinck : Littérature latine au Moyen Age, Paris, 1939, qui le tient lui-même de saint Anselme.

27. . Wahrheit und Methode, p. 77.

28. . Wahrheit und Methode, p. 166.

29. . Cf., Wahrheit und Methode. Ergänzungen. Register, Band 2.

30. . In : Deutsche Akademie für Sprache und Dichtung, Jahrbuch 1979, erste Lieferung, Heidelberg, Verlag Lambert Schneider, 1979, pp. 45-55.

31. . Cf., "Dichtung und Rhetorik im Mittelalter", in: E.R. Curtius : Gesammelte Aufsätze zur romanischen Philologie, Bern, Francke Verlag, 1950, 1984, p. 452.

32. . Wahrheit und Methode, p. 392-393.

33. . Wahrheit und Methode, p. 374.

34. . Conditions théoriques d'une histoire du sujet, p. 13.

35. . « Der junge Cocteau », in : KEEL, p. 390.

36. . Wahrheit und Methode, p. 169.

37. Cf. expression de Curtius dans son essai sur Dante et titre emprunté à Curtius par U. Raulff pour son article sur Curtius et l'Institut Warburg, paru le 12.10. 1989 dans la Frankfurter Allgemeine Zeitung.

\section{RÉSUMÉS}

Dans le sillage de l'herméneutique moderne, il apparaît que les travaux du philosophe $\mathrm{H}$. G. Gadamer et ceux du philologue E.R. Curtius accordent une place centrale à la réflexion sur la fonction de la littérature au $x^{\mathrm{e}}$ siècle. Les perspectives de l'aîné - Curtius -, et celle de son ancien élève - Gadamer -, bien que développées à une quinzaine d'années d'intervalle, semblent se 
rencontrer. L'objet du présent travail consiste à analyser cette rencontre, en montrant que la remise en cause des méthodes d'interprétation, le dépassement de l'esthétique issue de la modernité et le retour à la tradition rhétorique, unissent étroitement les deux chercheurs dans leur tentative d'éclairer les enjeux actuels de la pensée. Sans ignorer les différences, la question de la filiation de l'œuvre de Gadamer se trouve ainsi posée.

Im Zusammenhang mit der modernen Hermeneutik wird klar, dass die Forschungen des Philosophen H.G. Gadamer und des Philologen E.R. Curtius der Reflexion über die Funktion der Literatur im 20. Jahrhundert einen zentralen Stellenwert beimessen. Die Perspektiven des Älteren - Curtius -, sowie die seines ehemaligen Schülers - Gadamer -, scheinen sich zu begegnen, auch wenn sie im Abstand von etwa 15 Jahren entwickelt wurden. Die vorliegende Arbeit untersucht diese Begegnung, indem sie aufzeigt, wie die Infragestellung der Interpretationsmethoden, wie die Überwindung der Ästhetik aus der Zeit der Modernität und die Rückkehr zur rhetorischen Tradition, die beiden Gelehrten einen, in ihrem Versuch die aktuellen geistigen Fragen zu erläutern. Ohne die Unterschiede zu übersehen, wird schliesslich die Frage nach der geistigen Verwandtschaft des gadamerschen Werkes gestellt.

\section{AUTEUR}

\section{CHRISTINE JACQUEMARD-DE GEMEAUX}

Université de Clermont II 